\title{
Airway Management Evolution - In a Search for an Ideal Extraglottic Airway Device
}

\author{
Pavel Michálek ${ }^{1,2}$, Donald M. Miller ${ }^{3}$ \\ ${ }^{1}$ Department of Anaesthesiology and Intensive Care, First Faculty of Medicine, \\ Charles University in Prague and General University Hospital in Prague, Prague, \\ Czech Republic; \\ ${ }^{2}$ University of East Anglia, Norwich, United Kingdom; \\ ${ }^{3}$ Department of Anaesthetics, Guys Hospital, London, United Kingdom \\ Received June 19, 2014 ; Accepted November 18, 2014.
}

Key words: Extraglottic airway devices - Laryngeal mask airway - i-gel airway SLIPA - Cobra airway - Laryngeal tubes

\begin{abstract}
Extraglottic airway devices (EADs) are commonly used equipment for airway maintenance during elective procedures under general anaesthesia. They may be used also in other indications such as conduit for tracheal intubation or rescue airway device in prehospital medicine. Current classifications of the EADs lack systematic approach and therefore classification according to the sealing sites and sealing mechanisms is suggested in this review article. Modern EADs are disposable, latex-free devices made of plastic materials - most commonly from polyvinylchloride (PVC). The bowl of uncuffed sealers is manufactured from different materials such as thermoplastic elastomers or ethylene-vinyl-acetate co-polymer. EADs create various physical forces exerted on the adjacent tissues which may contribute to different sealing characteristic of particular device or to variable incidence of postoperative complications. Desired features of an ideal EAD involve easy insertion, high insertion success rate even by inexperienced users, protection against aspiration of gastric contents and low incidence of postoperative complications such as sore throat, hoarseness, cough or swallowing difficulties.
\end{abstract}

Mailing Address: Assoc. Prof. Pavel Michálek, MD., PhD., DESA, MSc., Department of Anesthesiology and Intensive Care, First Faculty of Medicine, Charles University in Prague and General University Hospital in Prague, $U$ nemocnice 2, 12021 Prague 2, Czech Republic; Phone: +420 224967 124; e-mail: pavel.michalek@vfn.cz 


\section{Introduction}

Extraglottic airway devices (EADs) - synonyms may be supraglottic airway devices (SADs, SGAs) or supralaryngeal airways (SLA) - are an integral part of modern anaesthetic practice. These devices are inserted into the oral cavity after induction to anaesthesia and help to provide patent airways during surgery. The EADs may also be used as a "rescue device" in emergency or difficult airway management. More than $50 \%$ of elective procedures conducted under general anaesthesia in the United Kingdom are managed using an EAD (Woodall and Cook, 2011). Their advantages include ease of insertion, high efficacy, and low incidence of postoperative complaints. They are a less invasive device than a tracheal tube (Brimacombe, 1995). First attempt insertion success rate is high even in hands of trainee anaesthesiologists or non-anaesthetic personnel (Ostermayer and GauscheHill, 2014).Various devices are available on the market at the moment. The first line EADs are derived from pharyngeal sealing devices such as the original Leech airway (Leech, 1937) and usually contain a proximal cuff for obstructing the pharynx and distal part allowing blockage or drainage of the upper part of the oesophagus. Second line devices are used more frequently in clinical settings and follow the shape of the original laryngeal mask airway (Brain, 1983) with modifications related to cuff design, presence or absence of additional channel for drainage of gastric contents, and internal mechanisms to decrease the incidence of epiglottic downfolding.

\section{Brief history of development of EADs}

Dr. Archibald lan Jeremy Brain is usually credited for the development of the first extraglottic airway device with peri-laryngeal sealing mechanism (Brain, 1983). However, Brain's laryngeal mask airway had its predecessors - pharyngeal inflatable extraglottic airway devices were used in clinical practice already before the World War II. A senior anaesthetist at Guy's Hospital in London, Dr. Francis Shipway, invented his pharyngeal airway in 1935, which featured an inflatable rubber balloon to prevent aspiration of blood during nasal procedures (Haridas, 2011). Two years later Dr. Beverley Charles Leech introduced a "pharyngeal bulb gasway" - an inflatable device mirroring pharyngeal and peri-laryngeal anatomy (Leech, 1937). Dr. Brain described his initial clinical experience with his device, named laryngeal mask airway (LMA), in 1983. This device was the first one encircling directly laryngeal structures and the end of its bowl was located very close to the vocal cords. The success of this device encouraged other inventors, and many other extraglottic airway devices have subsequently been invented since the 1990s. They may be divided to several groups according to the site of seal, sealing mechanisms, cuff location, and aspiration protection mechanisms such as the absence or presence of the additional tube for drainage of gastric contents. The development of laryngeal mask airways continued, with further devices including the flexible LMA designed for intraoral procedures (Brimacombe and Keller, 1999), 
ProSeal (Brain et al., 1995, 2000) and Supreme (Verghese and Ramaswamy, 2008) LMAs containing an additional gastric channel and intubating LMA (Fastrach) allowing blind intubation through the device in difficult airway scenarios (Baskett et al., 1998).Various other devices with a similar design to the LMA family were introduced in following years - AuraOnce, SoftSeal ${ }^{\circledR}$, LaPremiere or Solus laryngeal masks (Donaldson et al., 2011; Hernandez et al., 2012). Other extraglottic airway devices had different design than LMA family - including streamlined liner of pharyngeal airway (SLIPA) (Miller and Lavelle, 2002; Miller and Light, 2003), i-gel (Levitan and Kinkle, 2005; Michalek et al., 2013), Baska mask (Alexiev et al., 2012), Cobra Peri-laryngeal Airway (Hooshangi and Wong, 2008) or Laryngeal Tube (LT) and Laryngeal Tube Suction II (Asai and Shingu, 2005; Asai et al., 2007) (Table 1).

Table 1 - Main commercially available extraglottic airway devices divided according to our new proposed classification

\begin{tabular}{lll}
\hline Generation & Base-of-tongue (BT) sealers & Peri-laryngeal (PL) sealers \\
\hline $\begin{array}{l}\text { First generation } \\
\text { (inflatable }\end{array}$ & Combi-tube (+) (Covidien, & LMA Classic (-) (LMA Co., Seychelles) \\
mechanism & USA) & LMA Unique (-) (LMA Co., Seychelles) \\
of seal) & Rusch Easy Tube (+) & LMA Flexible (-) (LMA Co., Seychelles) \\
& (Teleflex, USA) & LMA Classic Excel (-) (I) (LMA Co., \\
& VBM Laryngeal Tube (-) & Seychelles) \\
& (VBM, Germany) & AuraOnce LM (-) (Ambu, Denmark) \\
& VBM LTS II (+) (VBM, & Aura-i LM (-) (I) (Ambu, Denmark) \\
& Germany) & Portex Soft Seal (-) (Smith Med., UK) \\
& King Laryngeal Tube (-) & Solus LM (-) (Intersurgical, UK) \\
& (King System, USA) & Sheridan LM (-) (Teleflex, USA) \\
& King LTS-D (+) (King & La Premiere Plus LM (-), LaEncore Plus \\
& System, USA) & LM (-) (Armstrong Medical, UK) \\
& Cobra PLA (-) (Pulmodyne, & Vital Seal LM (-) (GE Healthcare, USA) \\
& USA) & Ultra CPV (-) (AES, USA) \\
& Cobra Plus (-) (Pulmodyne, & Intubating LMA, Fastrach (-) (I) (LMA Co., \\
& USA) & Seychelles) \\
& & CTrach LMA (-) (I) (LMA Co., Singapore) \\
& & Air-Q ILA (-) (I) (Mercury Medical, USA) \\
& & LMA ProSeal (+) (LMA Co., Seychelles) \\
& & LMA Supreme (+) (LMA Co., Seychelles) \\
\hline Second generation \\
(wedge mechanism \\
of seal) & SLIPA (+) (CurveAir, UK) & i-gel (+) (I) (Intersurgical, UK) \\
\hline Third generation & S.A.T. (-) (I) (Ecolab, USA) & \\
mechanism of seal) & & Baska mask (+) (I) (Logikal \\
\hline & & Health Products, Australia) \\
\hline$(+)$ (-) & & \\
\hline
\end{tabular}

$(+)(-)$ - presence or absence of an additional channel for drainage of gastric contents or any mechanism for storage of regurgitated gastric contents; (I) - may be used as a conduit for an insertion of tracheal tube; LTS-D - laryngeal tube suction device; PLA - peri-laryngeal airway; LMA - laryngeal mask airway; LM - laryngeal mask; ILA - intubating laryngeal airway; SLIPA - Streamlined Liner of Pharyngeal Airway; S.A.L.T. - Supraglottic Airway Laryngopharyngeal Tube 
Some newer extraglottic airway devices as Aura-i (McAleavey and Michalek, 2010), Air-Q intubating laryngeal airway (Bakker et al., 2010) or CTrach LMA (Arslan et al., 2012) have been invented specifically to facilitate tracheal intubation in difficult airway scenarios.

\section{Classifications}

A consistent rational approach in the understanding of EADs is needed because there is a conflict of views that has led to inconsistent use of terminology.

The merit of Brimacombe's classification is that it is comprehensive but does not enhance understanding of the differences between groups (Brimacombe, 2004). Brimacombe recognizes three criteria: whether the EAD is cuffed or uncuffed, route of insertion (oral or nasal) and location of the cuff in relation to the hypopharynx. Cook's approach (Cook and Howes, 2011) sees EADs entirely from the LMA perspective and so suffers from being rather parochial. Hernandez has a rather mechanical structural classification which benefits from being consistent but lacks conceptual differences and again fails to aid understanding (Hernandez et al., 2012). Sealing site is a rational approach to distinguishing different EADs (Miller, 2004). Peri-laryngeal (PL) and Base-of-tongue (BT) sealing are the two sites that were identified (Miller, 2011). This approach is helpful in that it indicates the value of having two different sealing site EADs. Should one encounter failure of one device to seal, the use of another site EAD is more likely to act as an effective rescue airway rather than choosing another type with the same sealing site. A published case report, that has been repeated in practice many times, illustrates this point (Lim et al., 2012). The inconsistency with this early classification, before a variety of sealing mechanisms could be recognized however, mixed sealing site with sealing mechanism. In reality the SLIPA airway introduced a new sealing mechanism, wedge sealing. It fell into the group of Base-of-tongue sealing EADs. The original SLIPA prototype built in 1994 was made of soft silicone rubber and with its flexible walls had the characteristics of self-energizing sealing, which introduces yet a third sealing mechanism.

As the laryngeal mask, a peri-laryngeal sealing device, was established as the first line sealing extraglottic airway, it was clearly not the first EAD. Introducing the LMA (Brain, 1983), with patent priority date of December 1982, as the first generation and the later addition of a gastric tube in the ProSeal innovation (Brain et al., 1995) as the second generation device, can only be based upon an assumption that there is only one primary definitive supraglottic/extraglottic airway (Cook and Howes, 2011). The word "generation" intrinsically incorporates a meaning with a chronological component. According to the article that introduced this rather confusing terminology, devices designed with an aspiration protection mechanism are second generation devices. The precursor to the Combi-tube devised by Michael Frass (Frass et al., 1987), is a device invented by E.N. Scarberry (1980, US patent number 4231365) and Niemann (Niemann et al., 1984), with 
priority date January 1978, which, in turn, was derived from earlier pharyngeal sealing devices (Shipway, 1935 and Leech, 1937), so it actually predates the LMA by five to 65 years. So, instead of the terminology where the second generation was invented before the first generation device it makes more sense to reserve the word generation with real chronological meaning attached.

Sealing mechanism, in preference to a mechanism of aspiration protection would appear more logical (Miller, 2013). This is an improvement on an earlier classification of EADs that retains the importance of two different sealing sites (Miller, 2004).

First generation devices are those that use an inflating mechanism to seal that includes LMA and Combi-tube, both of which became available during the second half of the eighties decade. They represent two groups of devices with differing sealing sites, peri-laryngeal and base-of-tongue sealing devices.

Second generation devices are then devices that seal by wedging into position and rely upon elasticity of the pharyngeal tissues for sealing. The SLIPA airway (base-of-tongue sealing) appeared in 2002 and i-gel (peri-laryngeal sealing) about 3 years later.

Third generation devices would be self-energizing sealing devices. What is meant by self-energizing is that airway pressure itself is transmitted to the inside of a flexible sealing element so that as airway pressure rises, the airway pressure itself adds to the pressure of sealing against the mucosal wall thus maintaining a better seal when high inflation pressures are required. This achieves a variable sealing pressure against the mucosa, which may be very beneficial from a minimizing the risk of mucosal wall pressure damage. The Baska airway (2012) was possibly the first commercially available EAD having this characteristic (Alexiev et al., 2012).

Alternatively and in addition, we can use sealing site to classify EADs, there being two sites, peri-laryngeal sealing and base-of-tongue sealing. LMA is the classical peri-laryngeal sealer and Combi-tube or Laryngeal Tube is a base-of-tongue sealer (Table 1). So the miss-appropriately named Cobra Peri-laryngeal Airway (Cobra PLA) is actually a base-of-tongue sealer. Of the second generation sealing devices, the SLIPA is a base-of-tongue sealer and the i-gel is a peri-laryngeal sealer. Of the third generation sealing devices, there could be a base-of-tongue sealer (e.g. the developmental SLIPA in 1995 made of silicone had self-energizing sealing characteristics but was never produced commercially) but at the present, the only commercially available third generation devices are peri-laryngeal sealers such as the Baska airway (Alexiev et al., 2012).

\section{Material issues}

First prototypes of laryngeal mask airway were made from vulcanized rubber cuff taken from the Goldman nasal mask used for dental procedures in 1981 (Brain, 1983). Following prototypes of LMA cuff were created using different materials - medical grade silicone and latex. The silicone LMAs were reusable, 
allowing mechanical cleaning and sterilization. Latex-based devices were potentially dangerous due to a relatively high incidence of latex allergy $-1-2 \%$ in normal population but up to $10 \%$ among the healthcare professionals (Pollart et al., 2009). First disposable EAD was the LMA-Unique ${ }^{T M}$, made of the polyvinylchloride (PVC) (Verghese et al., 1998). Most available disposable laryngeal masks on the market, such as the Supreme LMA, AuraOnce or Solus LM, are made of the polyvinylchloride. SLIPA airway is manufactured from ethylene-vinyl-acetate copolymer which is a plastic material with limited flexibility. Although it was harder material, there were reported fewer sore throats despite it having more frequent traces of blood on the device (Lange et al., 2007). The bowl of the i-gel airway is made from a different material - very soft thermoplastic elastomer called styrene-ethylene-butadiene-styrene (SEBS) - and this is also different in design when compared with the LMA family because it does not need any inflation (Richez et al., 2008). Initially, it was expected that this material may warm up to body temperature and change its properties with subsequent improvement in peri-laryngeal seal. This speculation has not been confirmed in clinical practice (Nishiyama et al., 2012). SEBS is also very stable in high altitude and hyperbaric conditions which might create potential for its use during helicopter transfers and in diving medicine (Michalek et al., 2013). Devices providing pharyngeal or baseof-tongue seal are manufactured also from polyvinylchloride. The Cobra PLA ${ }^{\circledR}$ and Cobra PLUS contain PVC with different softness - a rigid head of device - and soft pharyngeal cuff (Hooshangi and Wong, 2008). The newest EAD, Baska mask is manufactured from a silicone (in its disposable version) and its medial soft cuff inflates proportionally with increasing inspiratory pressure while its lateral cuff is a self-recoiling membrane (Figure 1) (van Zundert and Gatt, 2012).

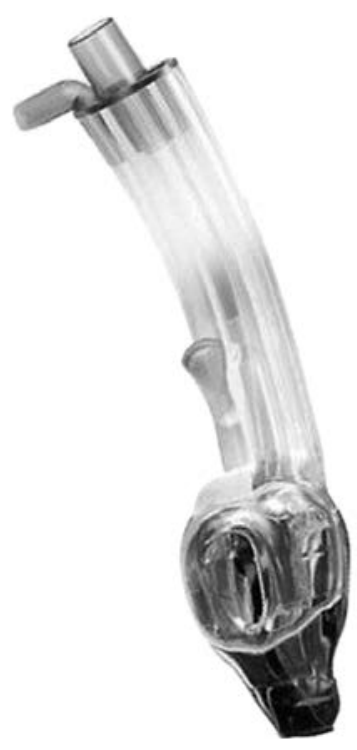

Figure 1 - Baska ${ }^{\circledR}$ mask - a novel extraglottic airway device with a self-energizing mechanism of sealing.

Michálek P.; Miller D. M. 
Common consensus regarding modern materials used for moulding EADs is that the devices should be latex-free (Demaegd et al., 2006) and, ideally, also phthalatefree.

\section{Single-use versus reusable devices}

Original EADs were manufactured as reusable equipment, requiring manual cleaning and sterilization after each clinical use. This situation changed in late 1990s. Concerns have been repeatedly raised about device cleanliness and safety after routine methods of sterilization. Miller et al. (2001) found significant amount of protein deposits on LMAs and other reusable anaesthetic airway equipment after routine cleaning which included chlorhexidine bath and autoclaving. Another study showed that protein cross-contamination occurs if used ProSeal LMAs are cleaned and autoclaved with unused devices (Richards et al., 2006). Protein deposits on the LMAs appear already after the first use of device and increase after each subsequent insertion (Greenwood et al., 2006). Most contamination is usually seen on the outer surface of the bowl as well as on its edges. The inner part of the cuff is relatively clean (Clery et al., 2003). How serious these fears are is very difficult to estimate because no case of prion infection transmission caused by reinforced extraglottic airway devices has been documented to date. High risk equipment for this type of transmission are reusable neurosurgical instruments, use of dural grafts and manipulations during the eye surgery (Laurenson et al., 1999; Hamaguchi et al., 2009). However, currently most manufacturers supply relatively cheap disposable extraglottic airway devices and only single-use devices are used in most developed countries.

\section{Physical forces exerted by the EADs}

Understanding some of the principles of physical forces that may be exerted when using EADs may be helpful in ascertaining the cause and preventing some of the side-effects encountered with different EADs (Miller, 2011). In addition, they may enable us to understand the variable performances as regards what makes for effective sealing.

As airway pressure rises, a pressure gradient is generated from inside to outside. This may constitute a force for expelling the device. Dislodgement of the device is likely to occur at the peak of inspiration. Factors that prevent that dislodgement are two-fold: Frictional forces and the direction of forces generated by the sealing mechanism in relation to the expulsive force (Miller, 2004). In the case of the LMA, the frictional force of the device being hooked around the base of the tongue is the main means of preventing it being expelled. The sealing forces related to the cuff are not perpendicular to the expulsive force as is the case with baseof-tongue sealing devices, which is mechanically advantageous. In the case of the ProSeal LMA, the addition of a cuff on the back of the peri-laryngeal seal corrects this imbalance to make it like the base-of-tongue sealers and makes for a more 
effective seal, where the expulsive forces are perpendicular to the sealing force (Miller, 2013).

Base-of-tongue sealers such as LT-D, LTSD (first generation sealers) and SLIPA (second generation sealing) seal with forces that are perpendicular to the expulsive force and hence they seal at higher inflation pressures (Miller and Camporota, 2006; Genzwuerker et al., 2007).

The standard LMA radius of curvature is greater than the natural curvature in the mouth as opposed to the Ambu Aura LM devices (Miller, 2013). Theoretically, this could result in a posterior force at the back of the pharynx so that if regurgitation occurs, the ascending liquid is more likely to enter the bowl of the LMA than to pass behind the LMA. The storage capacity of the LMA Unique is only $3.5 \mathrm{ml}$ which is at least 15 times less than in the SLIPA device (Miller, 2004), and therefore the risks of aspiration with regurgitation are theoretically higher. The use of positive end expiratory pressure or CPAP is likely to provide a measure of protection as it generates a positive pressure gradient (Russo et al., 2012). The posterior force against the pharyngeal wall because of the inadequate natural curvature of the LMA Unique will negate that effect. In the case of the i-gel, that will apply even more so but as regards the Ambu Aura-i with an accentuated natural curve, it should improve the situation. The need to accentuate the bend also increases the likelihood of the need for fixation of the device in position (Miller, 2011).

The same arguments are applicable to the i-gel and in combination with the nature of the seal at the entrance to the oesophagus; a lowering of confidence should be placed in the gastric tube to prevent aspiration. Associated with the increased force at the base of the tongue is obstruction of the venous drainage

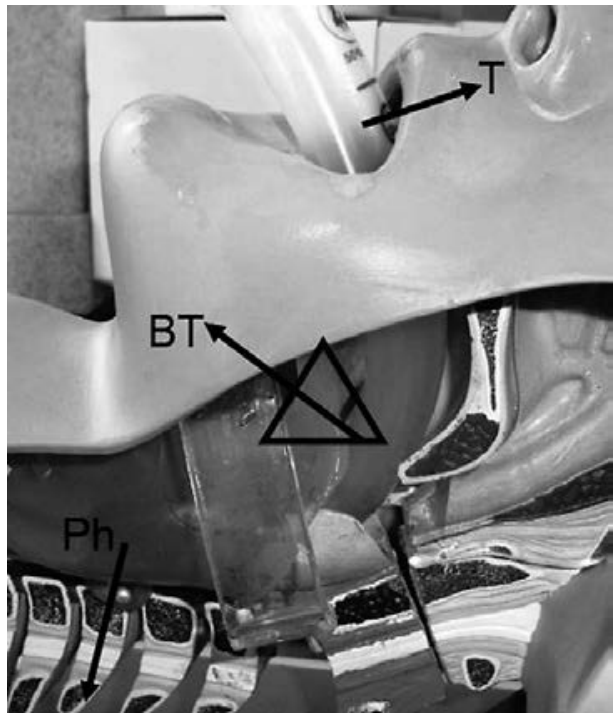

Figure 2 - The vectors of forces exerted on the adjacent tissues as a result of the i-gel bent in situ with a smaller radius of curvature than with which it is manufactured. " $T$ " demonstrates a force against the teeth, while "Ph" means a force against the posterior pharynx and "BT" represents the force against the tongue. The force $B T$ causes a pressure against the tongue which is expressed maximally at the pivotal point marked by the black triangle. This force may cause obstruction of the lingual venous drainage and is a possible explanation of a congested tongue finding with a rarely occurring associated loss of sensation at the tip of the tongue (reprinted from Miller D. (2013) Re-classification of extraglottic/supralaryngeal airway devices. In:The i-gel Supraglottic Airway, eds. Michalek P., Donaldson W., Nova Biomedical, New York). 
and lingual nerve damage is a likely explanation as to the occasional finding of a congested tongue (Figure 2) (Renes et al., 2011; Miller, 2013).

\section{Desired features of the EADs}

Factors motivating future development of EADs relate to the recorded actual and potential damage in the use of tracheal tubes during routine anaesthesia. An extensive meta-analysis involving two independent reviewers of 29 randomized prospective controlled trials - predetermined selection criteria, compared tracheal tube anaesthesia with anaesthesia using a laryngeal mask (Yu and Beirne, 2010). The findings are summarized in Table 2.

It is also noteworthy that after every case where tracheal tubes were used, there was an increase in resistance to airflow compared to when an EAD had been used (Tanaka et al., 2003).

This increase in resistance is attributed to swelling of laryngeal soft tissues.

Consistent with this finding is a more recent study (Yamanaka et al., 2009) which included over 3,000 routine cases that were intubated.

Figure 3 is a graphical redraw with a linear time scale of the findings in this study. This graphical display shows that there is a predictable injury profile with intubation.

Table 2 - Meta-analysis comparing the complications associated with the endotracheal tube vs. laryngeal mask airway (Yu and Beirne, 2010)

\begin{tabular}{lc}
\hline Complication & Risk ratio ETT/LMA \\
\hline Hoarse voice & 2.59 \\
Laryngospasm & 3.16 \\
Coughing & 7.12 \\
Sore throat & 1.67 \\
\hline
\end{tabular}

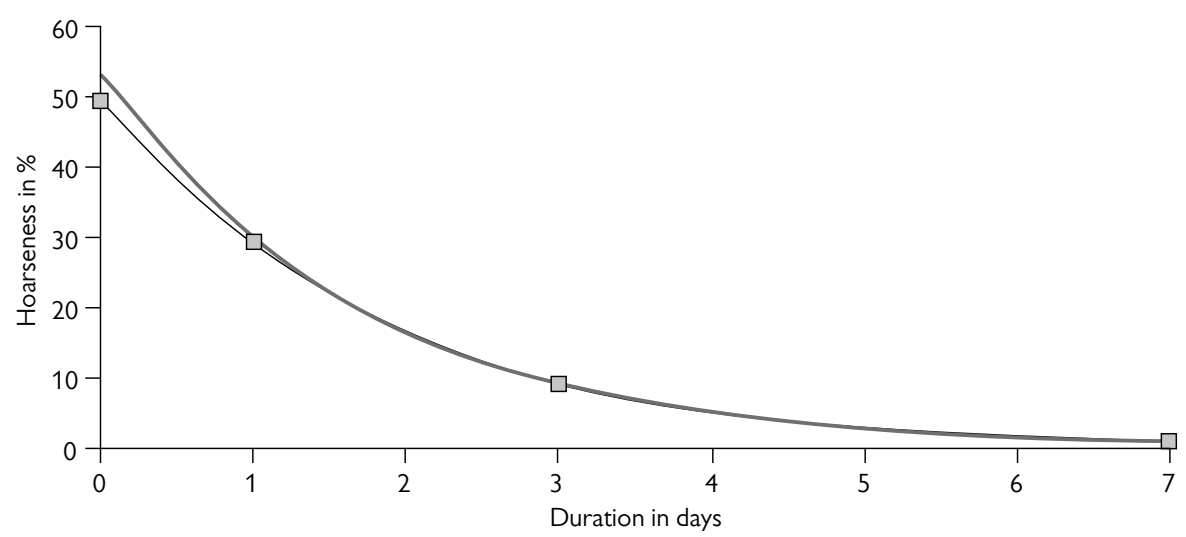

Figure 3 -The relationship between the incidence of hoarseness and time. The incidence of hoarseness was measured immediately after extubation, at 24 h, at 3 days and, finally, at 7 days (Yamanaka et al., 2009). 
The incidence of 1 per 1,000 cases with permanent hoarseness associated with arytenoid dislocation is quite an alarming figure. After cardiac surgery, the incidence is even more frequent with vocal cord dysfunction occurring in $1.9 \%$ of 270 cases of adult open heart surgery (Shafei et al., 1997).

There is one essential and two preferable requirements in any EAD (Miller, 2011). The essential requirement is good reliable sealing with a clear unobstructed airway (Miller, 2004). A greater magnitude of sealing pressure may be associated with greater risk of pressure damage if the sealing mechanism is a first generation sealing mechanism and, theoretically, is least if a third generation sealing mechanism is part of the design. The reason for this, is that self-energizing sealing should result in intermittent pressures as opposed to continuous pressure (Alexiev et al., 2012). This assumption sounds logical but has not been yet confirmed by any clinical trial.

The other two requirements are aspiration protection and the usefulness of the device for intubation with a tracheal tube (Miller, 2011). The latter may be important in the case of management of a difficult airway or in the case of resuscitation, where paramedics may not have had the opportunity of maintaining or learning intubation skills (Ostermayer and Gausche-Hill, 2014).

As regards aspiration protection, there are currently three aspiration protection mechanisms, namely, obstruction (e.g. a standard laryngeal mask airway obstructs the entrance to the oesophagus), drainage (e.g. the drainage tube in a ProSeal LMA or i-gel EAD) and storage (e.g. SLIPA which has a large capacity for storing liquids as in a water trap) (Miller, 2013). In the case of the latter, the effectiveness of the storage mechanism relates to the capacity and the position of the patient.

In the case of the drainage tube, its effectiveness relates to the nature and the position of the obstruction. Theoretically, an inflatable mechanism (e.g. ProSeal/ Supreme is likely to be more effective than a fixed volume device e.g. i-gel) (Schmidbauer et al., 2009). Sealing deeper in the oesophagus e.g. LTS II (LTSD) is likely to be more effective than at the entrance to the oesophagus e.g. ProSeal/ Supreme.

However, estimated risk of clinically significant aspiration associated with the EADs is extremely low - from $0.0009 \%$ (Cook et al., 2011) to $0.01-0.02 \%$ (Brimacombe and Berry, 1995; Bernardini and Natalini, 2009). On the contrary, regurgitation of gastric contents into the hypopharynx is much higher, occurring approximately in $5-10 \%$ of mechanically ventilated patients (Khazin et al., 2008).

As regards intubation or the use of a fibrescope, for accessing the respiratory system, this is dealt with more comprehensively in the relevant section of this article. However, it may be stated that while, this is rarely required, it may be a desirable characteristic in a routine device should this aspect be needed. The two situations where it may be needed are resuscitation by paramedics who do not have sufficient opportunity to keep their skills up-to-date and unanticipated difficult airway when one may need to use the EAD to facilitate intubation (Timmermann, 2011). 


\section{Clinical applications of the devices}

Extraglottic airway devices are the most commonly used airway maintenance devices during general anaesthesia in patients without increased risk for aspiration of gastric contents (Hernandez et al., 2012). This classical indication has been extended to other situations over last two decades. Laparoscopic surgery is now performed by many anaesthesiologists using an extraglottic airway. Some experts recommend using the devices with an incorporated additional channel for drainage or suctioning of gastric fluid in this instance (Drolet, 2009; Chen et al., 2013). ProSeal LMA, Supreme LMA or i-gel are devices allowing insertion of gastric tube for evacuation of gastric fluid (Drolet, 2009). SLIPA has a different mechanism separating respiratory and gastrointestinal tracts and allowing storage of gastric fluid, it being a hollow liner of the pharynx under pressure, thus providing a liquid trap within the device (Miller and Light, 2003). The devices with higher seal pressures such as ProSeal LMA, SLIPA, Supreme LMA or i-gel are also preferred in this situation because, mainly in gynaecological laparoscopies using Trendelenburg position, peak inspiratory pressures may result in the need for higher seal pressures tending to cause leaking around the devices (Genzwuerker et al., 2007; Cha et al., 2014). Some centres also insert EADs in the prone position (Brimacombe et al., 2007), although one can argue there would be difficult back-up plan in any case of complication or failure to ventilate.

Obese patients are another controversial indication for using of EADs. Several studies report successful airway maintenance during elective anaesthesia procedures using ProSeal LMA, Supreme LMA, SLIPA or i-gel airway (Natalini et al., 2003; Weber et al., 2011). Patients with mild (BMI > 30) or moderate (BMI > 35) obesity may tolerate these airways better than tracheal tube. A recent metaanalysis has shown that relevant data comparing tracheal tube and EADs in obese patients are available only for ProSeal LMA (Nicholson et al., 2013). Extraglottic airway devices may be used in obese patients as conduits for tracheal intubation -

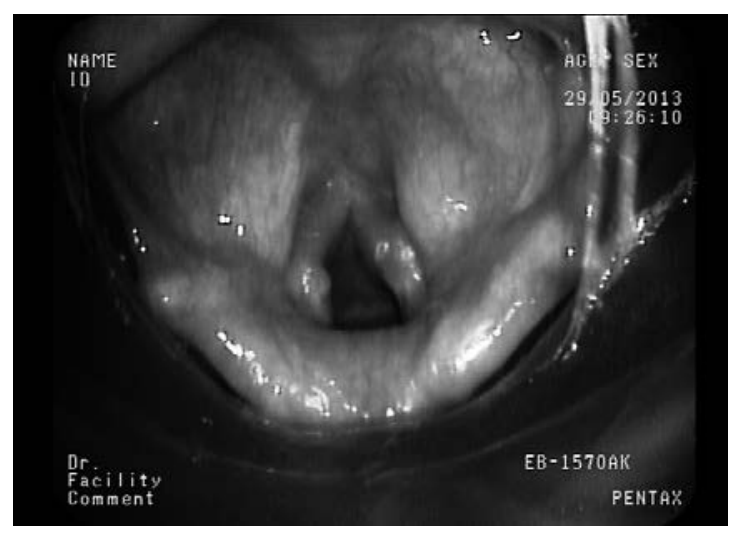

Figure 4 - Fibreoptic view through the correctly inserted i-gel airway. 
intubating LMA, CTrach LMA or i-gel (Figure 4) are suitable tools for insertion of tracheal tube through the device (Campbell et al., 2009; Arslan et al., 2012).

Laryngeal mask airway and intubating LMA (Fastrach ${ }^{\mathrm{TM}}$ ) are recommended in most difficult airway scenarios as "second line" devices in unexpected difficult or failed laryngoscopy (Henderson et al., 2004). Only the intubating LMA shows a high success rate - over $90 \%$ - for blind insertion of tracheal tube. Other extraglottic devices, such as the $\mathrm{i}-\mathrm{gel}$, air- $\mathrm{Q}$ intubating laryngeal airway or Aura-i are preferentially used as conduits for tracheal intubation with a flexible fibrescope because blind tube insertion through them has a low total success rate (Bakker et al., 2010; Theiler et al., 2011). EADs have also been extensively trialled in prehospital medicine (Ostermayer and Gausche-Hill, 2014). Their insertion is also recommended as a method of choice by paramedics and other healthcare professionals inexperienced in tracheal intubation in prehospital cardiac arrest and other conditions requiring tracheal intubation (Deakin et al., 2010). However, outcomes of patients whose airway were secured during out-of-hospital cardiac arrest with the LMA and other EADs are not better than in those intubated at scene or ventilated using bag-mask-valve (BMV) system (Tanabe et al., 2013).

\section{Extraglottic airway devices versus tracheal intubation}

Already mentioned systematic review, comparing laryngeal mask airways versus tracheal tube for airway maintenance in patients under general anaesthesia, found that LMAs have lower incidence of postoperative sore throat, hoarseness, coughing and reduced incidence of laryngospasm during recovery from anaesthesia (Yu and Beirne, 2010). Other parameters such as first attempt insertion success rate, nausea or vomiting did not differ between the devices. Whether EADs have lower incidence of aspiration of gastric contents is very difficult to validate due to an extremely low incidence of this complication - 0.009-0.02\% (Keller et al., 2004; Bernardini and Natalini, 2009). This systematic review focused only on some EADs - classical LMA, ProSeal LMA, flexible LMA, intubating LMA and SLIPA.A relatively large prospective study compared the incidence of aspiration of gastric contents between tracheal intubation and laryngeal mask airways in 65,712 patients (Bernardini and Natalini, 2009). The authors reported seven cases of aspiration associated with the tracheal tube and three cases with the laryngeal mask airways (LMA Classic and LMA Unique).

Patki performed a meta-analysis of published randomized controlled trials in paediatric population and found that laryngeal mask airways are superior to tracheal tube in respect of decreased incidence of cough during emergence, lesser postoperative sore throat and reduced incidence of vomiting (Patki, 2011). A disadvantage of the LMAs was the increased probability of failure to insert the device on the first attempt. The main limitation of this meta-analysis was that trials involving newer EADs as i-gel, AuraOnce or Supreme LMA were not included. 


\section{Conclusion}

Unlike the tracheal tube, whose design has not changed for decades, extraglottic airway devices are still developing and new prototypes and devices are being invented and extensively trialled. Most clinical studies relate to the LMA family, Cobra airway and the i-gel airway. Currently available EADs are widely used and have good safety profiles, but there are still some areas for improvement. Insertion in a small portion of patients - 5-10\% - fails due to anatomical differences in the peri-laryngeal area. That is why recognition of the two sealing sites may improve the success rate cutting the failure rate from $5 \%$ to less than $1 \%$ if the alternative sealing site was available. Epiglottis downfolding with a partial or total obstruction of the vocal cords is also sometimes present. The inflated cuff of the EADs may cause compression of the carotid arteries or ischaemia to the pharyngeal mucosa. A single gastric draining channel may get obstructed by stomach contents. The Baska ${ }^{\circledR}$ mask, the most novel EAD, has been invented with an effort to overcome most of these limitations - it contains broader gastric channels and a self-inflatable cuff. Unfortunately, its suggested superiority has not been so far confirmed in clinical practice (van Zundert and Gatt, 2012; Alexiev et al., 2013). So, the story continues and the ideal EAD which should have simple design, excellent seal, very high success rate of insertion on the first attempt even in hands of novices, reliable drainage mechanism, low incidence of postoperative discomfort and also low cost is still waiting to be invented.

\section{References}

Alexiev, V., Salim, A., Kevin, L. G., Laffey, J. G. (2012) An observational study of the Baska® mask: a novel supraglottic airway. Anaesthesia 67, 640-645.

Alexiev, V., Ochana, A., Abdelrahman, D., Coyne, J., McDonnell, J. G., O’Toole, D. P., Neligan, P., Laffey, J. G. (2013) Comparison of the Baska $\circledR$ mask with the single-use laryngeal mask airway in low-risk female patients undergoing ambulatory surgery. Anaesthesia 68, 1026-1032.

Arslan, Z.Y., Ozdamar, D., Yildiz, T. S., Solak, Z. M., Toker, K. (2012) Tracheal intubation in morbidly obese patients: a comparison of the Intubating Laryngeal Mask Airway ${ }^{\text {TM }}$ and Laryngeal Mask Airway CTrach $^{\text {TM }}$. Anaesthesia 67, 261-265.

Asai, T., Shingu, T. (2005) The laryngeal tube. Br. J. Anaesth. 95, 729-736.

Asai, T., Goy, R.W., Liu, E. H. (2007) Cricoid pressure prevents placement of the laryngeal tube and laryngeal tube-suction II. Br. J. Anaesth. 99, 282-285.

Bakker, J., Valkenburg, M., Galvin, E. M. (2010) Pilot study of the air-Q intubating laryngeal airway in clinical use. Anaesth. Intensive Care 38, 346-348.

Baskett, P. J., Parr, M. J., Nolan, J. P. (1998) The intubating laryngeal mask. Results of a multicentre trial with experience of 500 cases. Anaesthesia 53, 1174-1179.

Bernardini, A., Natalini, G. (2009) Risk of pulmonary aspiration with laryngeal mask airway and tracheal tube: analysis on 65712 procedures with positive pressure ventilation. Anaesthesia 64, 1289-1294.

Brain, A. I. J. (1983) The laryngeal mask - a new concept in airway management. Br. J. Anaesth. 55, 801-805.

Brain, A. I. J, Verghese, C., Strube, P., Brimacombe, J. (1995) A new laryngeal mask prototype - preliminary evaluation of seal pressures and glottic isolation. Anaesthesia 50, 42-48. 
Brain, A. I., Verghese, C., Strube, P. J. (2000) The LMA ProSeal - a laryngeal mask with an oesophageal vent. Br. J. Anaesth. 84, 650-654.

Brimacombe, J. (1995) The advantages of the LMA over the tracheal tube or facemask: a meta-analysis. Can. J. Anaesth. 42, 1017-1023.

Brimacombe, J. (2004) A proposed classification system for extraglottic airway devices. Anesthesiology 101, 559.

Brimacombe, J. R., Berry, A. (1995) The incidence of aspiration associated with the laryngeal mask airway: a meta-analysis of published literature. J. Clin. Anesth. 7, 297-305.

Brimacombe, J., Keller, C. (1999) A comparison of the flexible and standard laryngeal mask airways. Can.J. Anaesth. 46, 558-563.

Brimacombe, J. R., Wenzel, V., Keller, C. (2007) The ProSeal laryngeal mask airway in prone patients: a retrospective audit of 245 patients. Anaesth. Intensive Care 35, 222-225.

Campbell, J., Michalek, P., Deighan, M. (2009) I-gel supraglottic airway for rescue airway management and as a conduit for tracheal intubation in a patient with acute respiratory failure. Resuscitation 80, 963.

Cha, S. M., Park, S., Kang, H., Baek, C. W., Jung, Y. H., Cha, Y. J., In, J. (2014) Gastric distension with SLIPA versus LMA ProSeal during laparoscopic cholecystectomy: a randomized trial. Surg. Laparosc. Endosc. Percutan. Tech. 24, 216-220.

Chen, X., Jiao, J., Cong, X., Liu, L., Wu, X. (2013) A comparison of the performance of the i-gel ${ }^{\text {TM }}$ vs. the LMA-S ${ }^{\text {TM }}$ during anesthesia: a meta-analysis of randomized controlled trials. PLoS One 8, e71910.

Clery, G., Brimacombe, J., Stone, T., Keller, C., Curtis, S. (2003) Routine cleaning and autoclaving does not remove protein deposits from reusable laryngeal mask devices. Anesth. Analg. 97, 1189-1191.

Cook, T., Howes, B. (2011) Supraglottic airway devices: recent advances. Contin. Educ. Anaesth. Crit. Care Pain 11, 56-61.

Cook, T. M., Woodall, N., Frerk, C.; Fourth National Audit Project (2011) Major complications of airway management in the UK: results of the Fourth National Audit Project of the Royal College of Anaesthetists and the Difficult Airway Society. Part 1: anaesthesia. Br. J.Anaesth. 106, 617-631.

Deakin, C. D., Clarke, T., Nolan, J., Zideman, D. A., Gwinnutt, C., Moore, F., Ward, M., Keeble, C., Blancke, W. (2010) A critical reassessment of ambulance service airway management in prehospital care: Joint Royal Colleges Ambulance Liason Committee Airway Working Group, June 2008. Emerg. Med. J. 27, 226-233.

Demaegd, J., Soetens, F., Herregods, L. (2006) Latex allergy: a challenge for anaesthetists. Acta Anaesthesiol. Belg. 57, 127-135.

Donaldson, W., Abraham, A., Deighan, M., Michalek, P. (2011) i-gel ${ }^{T M}$ vs. AuraOnce ${ }^{T M}$ laryngeal mask for general anaesthesia with controlled ventilation in paralyzed patients. Biomed. Pap. Med. Fac. Univ. Palacky Olomouc Czech Repub. 155, 155-163.

Drolet, P. (2009) Supraglottic airways and pulmonary aspiration: the role of the drain tube. Can. J. Anaesth. 56, 715-720.

Frass, M., Frenzer, R., Zdrahal, F., Hoflehner, G., Porges, P., Lackner, F. (1987) The esophageal tracheal combitube: preliminary results with a new airway for CPR. Ann. Emerg. Med. 16, 768-772.

Genzwuerker, H.V., Altmayer, S., Hinkelbein, J., Gernoth, C., Viergutz, T., Ocker, H. (2007) Prospective randomized comparison of the new Laryngeal Tube Suction LTS II and the LMA-ProSeal for elective surgical interventions. Acta Anaesthesiol. Scand. 51, 1373-1377.

Greenwood, J., Green, N., Power, G. (2006) Protein contamination of the laryngeal mask airway and its relationship to re-use. Anaesth. Intensive Care 34, 343-346.

Hamaguchi, T., Noguchi-Shinohara, M., Nozaki, I., Nakamura, Y., Sato, T., Kitamoto, T., Mizusawa, H., Yamada, M. (2009) Medical procedures and risk for sporadic Creutzfeldt-Jakob disease, Japan, 1999-2008. Emerg. Infect. Dis. 15, 265-271.

Michálek P.; Miller D. M. 
Haridas, R. P. (2011) The Leech airway or pharyngeal bulb gasway. Anaesth. Intensive Care 39, S5-S10. Henderson, J. J., Popat, M. T., Latto, I. P., Pearce, A. C. (2004) Difficult Airway Society guidelines for management of the unanticipated difficult intubation. Anaesthesia 59, 675-694.

Hernandez, M. R., Klock, P.A. Jr., Ovassapian, A. (2012) Evolution of extraglottic airway: a review of its history, applications, and practical tips for success. Anesth. Analg. 114, 349-368.

Hooshangi, H., Wong, D. T. (2008) Brief review: the Cobra Peri-laryngeal Airway (Cobra PLA®) and Streamlined Liner of the Pharyngeal Airway $\left(\mathrm{SLIPA}^{\mathrm{TM}}\right)$ supraglottic airways. Can. J. Anaesth. 55, 177-185.

Keller, C., Brimacombe, J., Bittersohl, J., Lirk, P., von Goedecke, A. (2004) Aspiration and the laryngeal mask airway: three cases and a review of the literature. Br. J. Anaesth. 93, 579-582.

Khazin, V., Ezri, T., Yishai, R., Sessler, D. I., Serour, F., Szmuk, P., Evron, S. (2008) Gastroesophageal regurgitation during anesthesia and controlled ventilation with six airway devices. J. Clin. Anesth. 20, 508-513.

Lange, M., Smul, T., Zimmermann, P., Kohlenberger, R., Roewer, N., Kehl, F. (2007) Effectiveness and patient comfort of the novel Streamlined Pharynx Airway Liner (SLIPA) compared with the conventional Laryngeal Mask Airway in opthalmic surgery. Anesth. Analg. 104, 431-434.

Laurenson, I. F., Whyte, A. S., Fox, C., Babb, J. R. (1999) Contaminated surgical instruments and variant Creutzfeldt-Jakob disease. Lancet 354, 1823-1824.

Leech, B. C. (1937) The pharyngeal bulb gasway: a new aid in cyclopropane anesthesia. Curr. Res. Anesth. Analg. 16, 22-25.

Levitan, R. M., Kinkle,W. C. (2005) Initial anatomic investigation of the i-gel airway: a novel supraglottic airway without inflatable cuff. Anaesthesia 60, 1022-1026.

Lim, B. G., Kim, K. J., Kong, M. H., Kim, N. S., Lim, S. H., Lee, M. K., Lee, I. O. (2012) SLIPA ${ }^{T M}$ may be more useful than other LMADs for difficult airway management. Can. J. Anesth. 59, 120-121.

McAleavey, F., Michalek, P. (2010) Aura-i laryngeal mask as a conduit for elective fibreoptic intubation. Anaesthesia 65, 1151.

Michalek, P., Donaldson, W., Theiler, L. (2013) The use of the i-gel in anaesthesia - Facts and fiction in 2013. Trends in Anaesthesia and Critical Care 3, 246-251.

Miller, D. M. (2004) A proposed classification and scoring system for supraglottic sealing airways: a brief review. Anesth. Analg. 99, 1553-1559.

Miller, D. M. (2011) A conceptual ideal supraglottic airway. Anest. Intenziv. Med. 22, 149-152.

Miller, D. M. (2013) Re-classification of extraglottic/supralaryngeal airway devices. In: The i-gel Supraglottic Airway, eds. Michalek, P., Donaldson, W., pp. 15-28, Nova Biomedical, New York.

Miller, D. M., Lavelle, M. (2002) A Streamlined Pharynx Airway Liner: pilot study in 22 patients in controlled and spontaneous ventilation. Anesth. Analg. 94, 759-761.

Miller, D. M., Light, D. (2003) Laboratory and clinical comparisons of the Streamlined Liner of the Pharynx Airway (SLIPA) with the laryngeal mask airway. Anaesthesia 58, 136-142.

Miller, D. M., Camporota, L. (2006) Advantages of ProSeal and SLIPA airways over tracheal tubes for gynecological laparoscopies. Can. J. Anaesth. 53, 188-193.

Miller, D. M., Youkhana, I., Karunaratne, W. U., Pearce, A. (2001) Presence of protein deposits on cleaned reusable anaesthetic equipment. Anaesthesia 56, 1069-1072.

Natalini, G., Franceschetti, M. E., Pantelidi, M.T., Rosano, A., Lanza, G., Bernardini, A. (2003) Comparison of the standard laryngeal mask airway and the ProSeal laryngeal mask airway in obese patients. Br. J. Anaesth. 90, 323-326.

Nicholson, A., Cook, T. M., Smith, A. F., Lewis, S. R., Reed, S. S. (2013) Supraglottic airway devices versus tracheal intubation for airway management during general anaesthesia in obese patients. Cochrane Database Syst. Rev. 9, CD010105. 
Niemann, J.T., Rosborough, J. P., Myers, R., Scarberry, E. N. (1984) The pharyngo-tracheal lumen airway: preliminary investigation of a new adjunct. Ann. Emerg. Med. 13, 591-596.

Nishiyama, T., Kohno, Y., Kim, H. J., Shin, W. J., Yang, H. S. (2012) The effects of pre-warming the i-gel on fitting to laryngeal structure. Am. J. Emerg. Med. 30, 1756-1759.

Ostermayer, D. G., Gausche-Hill, M. (2014) Supraglottic airways: the history and current state of prehospital airway adjuncts. Prehosp. Emerg. Care 18, 106-115.

Patki, A. (2011) Laryngeal mask airway vs. the endotracheal tube in paediatric airway management: a metaanalysis of prospective randomised controlled trials. Indian J. Anaesth. 55, 537-541.

Pollart, S. M., Warniment, C., Mori, T. (2009) Latex allergy. Am. Fam. Physician 80, 1413-1418.

Renes, S. H., Zwart, R., Scheffer, G. J., Renes, S. (2011) Lingual nerve injury following the use of an i-gel laryngeal mask. Anaesthesia 66, 226-227.

Richards, E., Brimacombe, J., Laupau, W., Keller, C. (2006) Protein cross-contamination during batch-cleaning and autoclaving of the ProSeal ${ }^{\mathrm{TM}}$ laryngeal mask airway. Anaesthesia 61, 431-433.

Richez, B., Saltel, L., Banchereau, F., Torrielli, R., Cros, A. M. (2008) A new single use supraglottic airway device with a non-inflatable cuff and an esophageal vent: an observational study of the i-gel. Anesth. Analg. 106, 1137-1139.

Russo, S. G., Cremer, S., Mühlhäuser, U., Eich, C., Quintel, M., Bauer, M. (2012) Investigating the fluid seal of supraglottic airway devices in humans using indicator dye via the drainage tube: a potential roadmap for future studies. Open Journal of Anesthesiology 2, 18-22.

Schmidbauer,W., Bercker, S., Volk, T., Bogusch, G., Mager, G., Kemer,T. (2009) Oesophageal seal of the novel supralaryngeal device i-gel in comparison with the laryngeal mask airways Classic and ProSeal using a cadaver model. Br. J. Anaesth. 102, 135-139.

Shafei, H., el-Kholy, A., Azmy, S., Ebrahim, M., al-Ebrahim, K. (1997) Vocal cord dysfunction after cardiac surgery: an overlooked complication. Eur. J. Cardiothorac. Surg. 11, 564-566.

Shipway, F. (1935) Airway for intranasal operations. Br. Med.J. 1, 767.

Tanabe, S., Ogawa, T., Akahane, M., Koike, S., Horiguchi, H., Yasunaga, H., Mizoguchi, T., Hatanaka, T., Yokota, H., Imamura, T. (2013) Comparison of neurological outcome between tracheal intubation and supraglottic airway device insertion of out-of-hospital cardiac arrest patients: a nationwide, population-based, observational study. J. Emerg. Med. 44, 389-397.

Tanaka, A., Isono, S., Ishikawa, T., Sato, J., Nishino, T. (2003) Laryngeal resistance before and after minor surgery: endotracheal tube versus Laryngeal Mask Airway. Anesthesiology 99, 252-258.

Theiler, L., Kleine-Brueggeney, M., Urwyler, N., Graf, T., Luyet, C., Greif, R. (2011) Randomized clinical trial of the i-gel ${ }^{T M}$ and Magill tracheal tube or single-use ILMA ${ }^{T M}$ and ILMA ${ }^{T M}$ tracheal tube for blind intubation in anaesthetised patients with a predicted difficult airway. Br. J. Anaesth. 107, 243-250.

Timmermann, A. (2011) Supraglottic airways in difficult airway management: successes, failures, use and misuse. Anaesthesia 66, S45-S56.

van Zundert, T., Gatt, S. (2012) The Baska Mask $\AA_{-}$- a new concept in self-sealing membrane cuff extraglottic airway devices, using a sump and two gastric drains: a critical evaluation. J. Obstet. Anaesth. Crit. Care $\mathbf{2}$, 23-30.

Verghese, C., Ramaswamy, B. (2008) LMA Supreme - a new single-use LMA with gastric access: a report on its clinical efficacy. Br. J. Anaesth. 101, 405-410.

Verghese, C., Berlet, J., Kapila, A., Pollard, R. (1998) A clinical assessment of the single use laryngeal mask airway the LMA-Unique ${ }^{T M}$. Br. J. Anaesth. 80, 677-679.

Weber, U., Oguz, R., Potura, L. A., Kimberger, O., Kober, A., Tschernko, E. (2011) Comparison of the i-gel and LMA-Unique laryngeal mask airway in patients with mild to moderate obesity during elective shortterm surgery. Anaesthesia 66, 481-487. 
Woodall, N. M., Cook, T. M. (2011) National census of airway management techniques used for anaesthesia in the UK: first phase of the Fourth National Audit Project at the Royal College of Anaesthetists. Br. J. Anaesth. 106, 266-271.

Yamanaka, H., Hayashi, Y., Watanabe, Y., Uematu, H., Mashimo, T. (2009) Prolonged hoarseness and arytenoid cartilage dislocation after tracheal intubation. Br. J. Anaesth. 103, 452-455.

Yu, S. H., Beirne, O. R. (2010) Laryngeal mask airways have a lower risk of airway complications compared with endotracheal intubation: a systematic review. J. Oral Maxillofac. Surg. 68, 2359-2376. 